\title{
Overexpression of GRP75 inhibits inflammation in a rat model of intracerebral hemorrhage
}

\author{
LIAN-JIE LV, JIA LI, HAI-BO QIAO, BEN-JIN NIE, PENG LU, FENG XUE and ZHI-MING ZHANG \\ Department of Neurosurgery, Tianjin Nankai Hospital, Tianjin 300100, P.R. China
}

Received November 22, 2015; Accepted November 15, 2016

DOI: $10.3892 / \mathrm{mmr} .2017 .6126$

\begin{abstract}
Glucose-regulated protein 75 (GRP75) is a member of the heat shock protein 70 family and previous studies have demonstrated that GRP75 is involved in diseases of the central nervous system. However, the biological function of GRP75 in intracerebral hemorrhage $(\mathrm{ICH})$ remains to be clarified. Thus, the aim of the present study was to evaluate the effects of GRP75 in a rat model of ICH. Western blotting was used to detect the protein expression of GRP75, active caspase-3, Bax, Bcl-2, p-Akt and Akt in brain tissues following ICH. The levels of tumor necrosis factor- $\alpha$ (TNF- $\alpha$ ) and interleukin (IL)- $1 \beta$ were evaluated using ELISA assay. Expression of GRP75 mRNA and protein was demonstrated to be reduced in the brain tissues of rats with ICH compared with sham-operated rats. In addition, overexpression of GRP75 in brain tissues with ICH significantly inhibited the production of the inflammatory cytokines TNF- $\alpha$ and IL-1 $\beta$ and increased Bcl-2/decreased Bax levels compared with ICH alone. Furthermore, overexpression of GRP75 in brain tissues with ICH resulted in significantly increased phosphorylation of Akt compared with ICH alone. Therefore, the present study demonstrated, for the first time to the best of our knowledge, significantly reduced GRP75 expression in brain tissues following $\mathrm{ICH}$, and that overexpression of GRP75 inhibits inflammation and potentially inhibits neuronal apoptosis in a rat model of ICH. GRP75 may, therefore, represent a promising target in the treatment of $\mathrm{ICH}$.
\end{abstract}

\section{Introduction}

Intracerebral hemorrhage ( $\mathrm{ICH})$, estimated to affect over 1 million people worldwide each year, accounts for $10-15 \%$ of all strokes and is associated with high mortality and morbidity (1). To date, no effective therapeutic strategy exists to improve the quality of life of patients with $\mathrm{ICH}$. ICH is

Correspondence to: Dr Zhi-Ming Zhang, Department of Neurosurgery, Tianjin Nankai Hospital, 102 Sanwei Road, Tianjin 300100, P.R. China

E-mail: zhang_zzhm@163.com

Key words: glucose-regulated protein 75, intracerebral hemorrhage, inflammation, neuron apoptosis a rapidly evolving process that causes necrosis, followed by apoptotic cell death and altered gene expression in the surrounding brain tissue (2). Thus, the identification of novel therapeutic agents for preventing ICH is crucial.

Glucose-regulated protein 75 (GRP75), a member of the heat shock protein 70 family, is located in multiple organelles, but predominantly in mitochondria (3). A growing body of evidence has demonstrated the role of GRP75 in regulating cellular stress responses, mitochondrial homeostasis, intracellular trafficking, antigen presenting, cell proliferation, differentiation, and tumorigenesis (4). A potential protective effect of GRP75 has also been demonstrated towards diseases of the central nervous system. Zhang et al (5) reported that GRP75 was implicated in the process of B cell lymphoma 2 apoptosis regulator ( $\mathrm{Bcl}-2)$ - and caspase-dependent apoptosis of retinal ganglion cells (RGCs) following optic nerve crush. Overexpression of GRP75 in PC12 cells has also been demonstrated to prevent apoptosis by reducing the expression of $\mathrm{Bcl}-2$ associated $\mathrm{X}$ apoptosis regulator (Bax) and delaying the release of cytochrome $c$ (6). In addition, overexpression of GRP75 attenuates the lipopolysaccharide-induced oxidative and metabolic responses, and suppresses the inflammatory response in microglial BV-2 cells (7). However, to date, the biological function of GRP75 in ICH remains unclear. Thus, the purpose of the present study was to evaluate the effects of GRP75 in a rat model of ICH. A distinct neuroprotective strategy has been identified that prevents an inflammatory response and neuron apoptosis following $\mathrm{ICH}$.

\section{Materials and methods}

Animals and rat model of ICH. All animals were purchased from the laboratory animal center of Tianjin Nankai Hospital (Tianjin, China). Sixty 5 week old adult male Sprague-Dawley rats, with body weight of 350-400 g were maintained at constant temperature $\left(21 \pm 2^{\circ} \mathrm{C}\right)$ and humidity in a holding facility under a 12-h light-dark cycle, with free access to food and water. Animal experiments conformed to the guidelines issued by Tianjin Nankai Hospital for laboratory animals. Rats were anesthetized intraperitoneally with sodium pentobarbital $(50 \mathrm{mg} / \mathrm{kg})$. Anesthetized rats were placed in a stereotactic apparatus (David Kopf Instruments, Tujunga, CA, USA). A 26-gauge needle was then implanted into the caudate-putamen at the following coordinates: $0.8 \mathrm{~mm}$ posterior, $5.5 \mathrm{~mm}$ ventral, $3.5 \mathrm{~mm}$ lateral to the bregma (8). ICH was induced 
with an infusion of $0.7 \mu \mathrm{l}$ saline and $0.14 \mathrm{U}$ collagenase IV (Sigma-Aldrich; Merck Millipore, Darmstadt, Germany) by using a microinfusion pump (Harvard Apparatus, Holliston, MA, USA) over a duration of $7 \mathrm{~min}$. Rats with sham surgery were received $0.7 \mu 1$ saline only.

Reverse transcription-quantitative polymerase chain reaction $(R T-q P C R)$ analysis. Total RNA was extracted from the whole brain tissues using the RNA plus kit (Fermentas; Thermo Fisher Scientific, Inc., Waltham, MA, USA). Reverse transcription of $5 \mu \mathrm{g}$ of the total RNA into cDNA was performed using M-MLV reverse transcriptase (Clontech Laboratories, Inc., Mountainview, CA, USA). The levels of mRNA transcripts were analyzed by qPCR using Power SYBR-Green PCR Master Mix (Applied Biosystems; Thermo Fisher Scientific, Inc.) on an ABI Prism 7500 sequence detector (Applied Biosystems; Thermo Fisher Scientific, Inc.). The gene-specific primer sequences used for detection of GRP75 were 5'-CGGCTACCACATCCAAGGAA-3' (forward) and 5'-GCTCGAATTACCGCGGCT-3' (reverse), and for $\beta$-actin were 5'-AAATCGTGCGTGACATCAAAGA-3' (forward), and 5'-GGCCATCTCCTGCTCGAA-3' (reverse). Thermocycling conditions were as follows: $94^{\circ} \mathrm{C}$ for $4 \mathrm{~min}, 94^{\circ} \mathrm{C}$ for $20 \mathrm{sec}$, $58^{\circ} \mathrm{C}$ for $30 \mathrm{sec}$ and $72^{\circ} \mathrm{C}$ for $20 \mathrm{sec}, 2 \mathrm{sec}$ for plate reading for 40 cycles and melting curve from 65 to $95^{\circ} \mathrm{C}$. $\beta$-actin was used as a quantitative and qualitative control to normalize the gene expression. Data were analyzed by the $2^{-\Delta \Delta \mathrm{Ct}}$ method (9).

Western blotting. Total protein extracts were prepared from the whole brain using radioimmunoprecipitation assay lysis buffer (Beyotime Institute of Biotechnology, Haimen,China) according to the manufacturer's protocol. The protein concentration in the lysates was evaluated using a bicinchoninic acid protein assay kit (Beyotime Institute of Biotechnology). For protein separation, a total of $20 \mu \mathrm{g}$ of protein was loaded on a $12.5 \%$ sodium dodecyl sulfate gel and separated by polyacrylamide gel electrophoresis, followed by transfer to a nitrocellulose membrane (Bio-Rad Laboratories, Inc., Hercules, CA, USA). Membranes were subsequently blocked with $2.5 \%$ non-fat milk for $1 \mathrm{~h}$ at $37^{\circ} \mathrm{C}$, then probed with anti-rabbit GRP75 (1:3,000; catalog no. sc-13967), caspase 3 (1:3,000; catalog no. sc-98785), Bcl-2 (1:2,500; catalog no. sc-783), Bax (1:2,500; catalog no. sc-6236), Akt (1:2,500; catalog no. sc-24500), p-Akt (1:2,500; catalog no. sc-135650) and GAPDH (1:1,500; catalog no. sc-367714). The primary antibodies were obtained from Santa Cruz Biotechnology, Santa Cruz, CA, USA) and incubation occurred overnight at $4^{\circ} \mathrm{C}$. The membrane was then incubated with a chicken anti-rabbit horseradish peroxidase-conjugated secondary antibody (1:30,2,500; catalog no. sc-516087; Santa Cruz Biotechnology) diluted in blocking buffer for $1 \mathrm{~h}$ at room temperature. Blots were developed using an enhanced chemiluminescence system (Pierce; Thermo Fisher Scientific, Inc.). Protein expression was analyzed using BandScan 5.0 software (Glyko Biomedical Ltd., Novato, CA, USA).

GRP75 expression vector and in vivo injection. The cDNA encoding GRP75, obtained from PCR amplification, was amplified and subcloned into the adenoviral shuttle vector pAd-CMV (Invitrogen; Thermo Fisher Scientific, Inc.). Rat green fluorescent protein (GFP; Invitrogen, Thermo Fisher
Scientific, Inc.) was used as a non-specific control. The adenoviral shuttle vector pAd-CMV and adenoviral gene expression vector pAdEasy-1 were recombined in Escherichia coli strain BJ5183. The recombined plasmid Ad-GRP75 was then propagated in 293T cells (American Type Culture Collection, Manassas, VA, USA). For in vivo injection, $5 \mu 1$ of Ad-GFP or Ad-GRP75 dissolved in $30 \mu \mathrm{l}$ i-Fect transfection reagent (Neuromics, Edina, MN, USA) was administered intrathecally for 1 day, from 1 day prior to ICH and rats were sacrificed 2 days subsequent to $\mathrm{ICH}$ for analysis of neuron apoptosis.

Enzyme-linked immunosorbent assay (ELISA). The brain tissues were rapidly removed and homogenized in lysis buffer. Following centrifugation $(6000 \times \mathrm{g})$ at $4^{\circ} \mathrm{C}$ for $10 \mathrm{~min}$, the supernatants were analyzed for cytokine production by ELISA. ELISA was performed as per the manufacturer's instructions (Dakewe Biotech Co., Ltd., Shenzhen, China) to assess the concentrations of tumor necrosis factor- $\alpha$ (TNF- $\alpha$; catalog no. DKW12-2720-048) and interleukin (IL)-1 $\beta$ (catalog no. DKW12-2012-096) in the culture supernatant.

Statistical analysis. Data was expressed as the mean \pm standard deviation. Statistical analysis was performed with SPSS software version 13 (SPSS, Inc., Chicago, IL, USA). Multiple comparisons were evaluated by one-way analysis of variance followed by Tukey's post hoc test. Two-group comparisons were analyzed by Student's $t$ test. $\mathrm{P}<0.05$ was considered to indicate a statistically significant difference.

\section{Results}

Expression of GRP75 in a rat model of ICH. GRP75 expression was investigated in brain tissues at different time points subsequent to ICH. Compared with the sham-operated group, GRP75 mRNA expression levels were significantly decreased from $6 \mathrm{~h}$ following $\mathrm{ICH}(\mathrm{P}=0.038$; Fig. 1A), with progressive decreases at subsequent time points, to a minimum level at 2 days $(\mathrm{P}=0.014$; Fig. 1A). Furthermore, western blot analysis demonstrated that GRP75 protein expression levels were also significantly inhibited by $\mathrm{ICH}$ in the same progressive manner (Fig. 1B).

Overexpression of GRP75 inhibits the inflammatory response in a rat model of ICH. To investigate the role of GRP75 in the progression of ICH, $5 \mu \mathrm{l}$ of Ad-GFP or Ad-GRP75 dissolved in $30 \mu \mathrm{l}$-Fect transfection reagent (Neuromics, Edina, MN, USA) was administered intrathecally once daily for 1 days. Subsequent GRP75 expression levels were determined by RT-qPCR and western blot. GRP75 mRNA and protein expression levels were confirmed to be significantly increased in brain tissues that received Ad-GRP75 compared with tissues in the Ad-GFP control group $(\mathrm{P}=0.021$ and $\mathrm{P}=0.036$; Fig. $2 \mathrm{~A}$ and $\mathrm{B}$, respectively). In addition, overexpression of GRP75 in brain tissues of rats with ICH significantly suppressed the production of TNF- $\alpha$ and IL-1 $\beta$ compared with the ICH group $(\mathrm{P}=0.026$ and $\mathrm{P}=0.032$; Fig. $3 \mathrm{~A}$ and $\mathrm{B}$, respectively).

Overexpression of GRP75 inhibits expression of neuronal apoptosis markers in a rat model of ICH. Neuronal apoptosis is a significant event surrounding hematoma in ICH (10). 

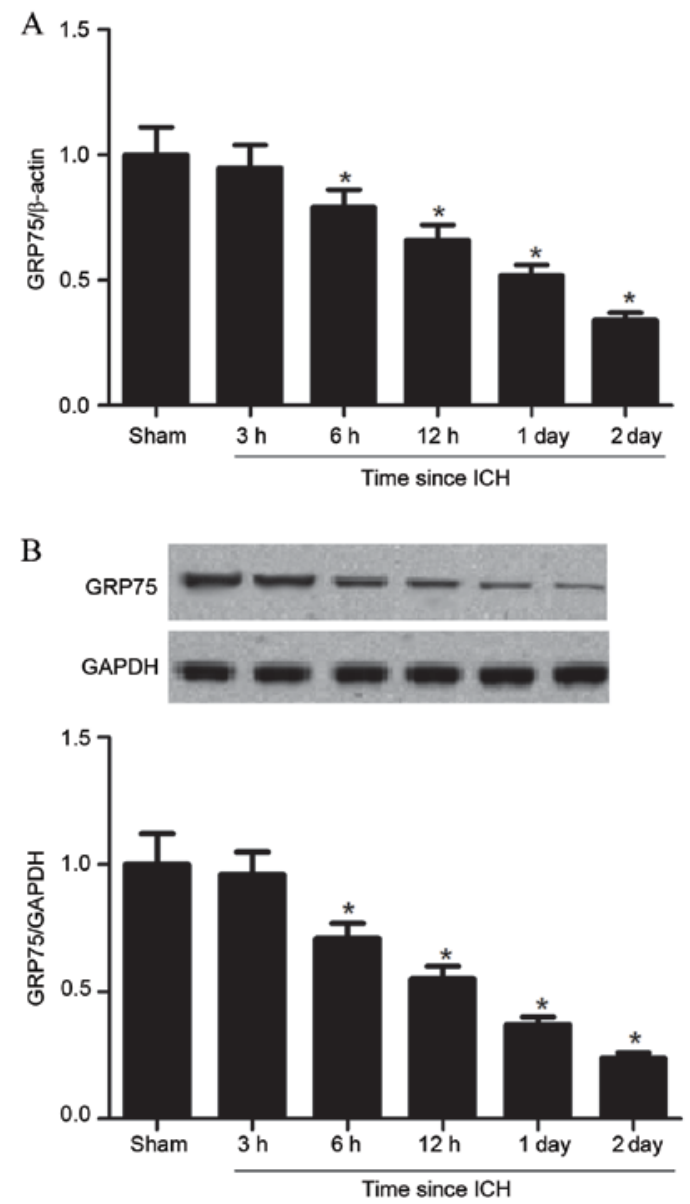

Figure 1. Expression of GRP75 in a rat model of ICH. (A) Reverse transcription-quantitative polymerase chain reaction analysis was performed to determine GRP75 mRNA expression levels in brain tissues at different time periods following ICH, relative to $\beta$-actin control. (B) Western blot analysis was performed to determine GRP75 protein expression levels in brain tissues at different time periods following ICH. Protein expression was normalized to GAPDH. Values are presented as the mean \pm standard deviation. $n=5$ rats per group. " $\mathrm{P}<0.05$ vs. sham group. GRP75, glucose-regulated protein 75 ; $\mathrm{ICH}$, intracerebral hemorrhage.

The effects of GRP75 on neuronal apoptosis were, therefore, investigated in a rat model of ICH. Active caspase-3 protein expression was increased following ICH compared with the sham group ( $\mathrm{P}=0.019$; Fig. 4), while overexpression of GRP75 in brain tissues of rats with ICH significantly inhibited the expression of active caspase- 3 compared with the ICH group ( $\mathrm{P}=0.041$; Fig. 4). To further examine the effects of GRP75 on neuronal apoptosis, the expression profiles of Bax and Bcl-2 were investigated. Overexpression of GRP75 in brain tissues of rats with ICH significantly decreased the expression of Bax and increased the expression of Bcl-2 compared with the ICH group $(\mathrm{P}=0.023$ and $\mathrm{P}=0.036$ respectively; Fig. 4).

Overexpression of GRP75 upregulates the level of $p$-Akt in a rat model of $\mathrm{ICH}$. To examine whether GRP75 promotes neuronal apoptosis via the PI3K/Akt signaling pathway, the effect of GRP75 overexpression on Akt phosphorylation was examined. The level of Akt Ser473 phosphorylation was demonstrated to be significantly diminished 2 days subsequent to ICH compared with the sham group ( $\mathrm{P}=0.017$; Fig. 5), whereas overexpression of GRP75 in brain tissues of rats with
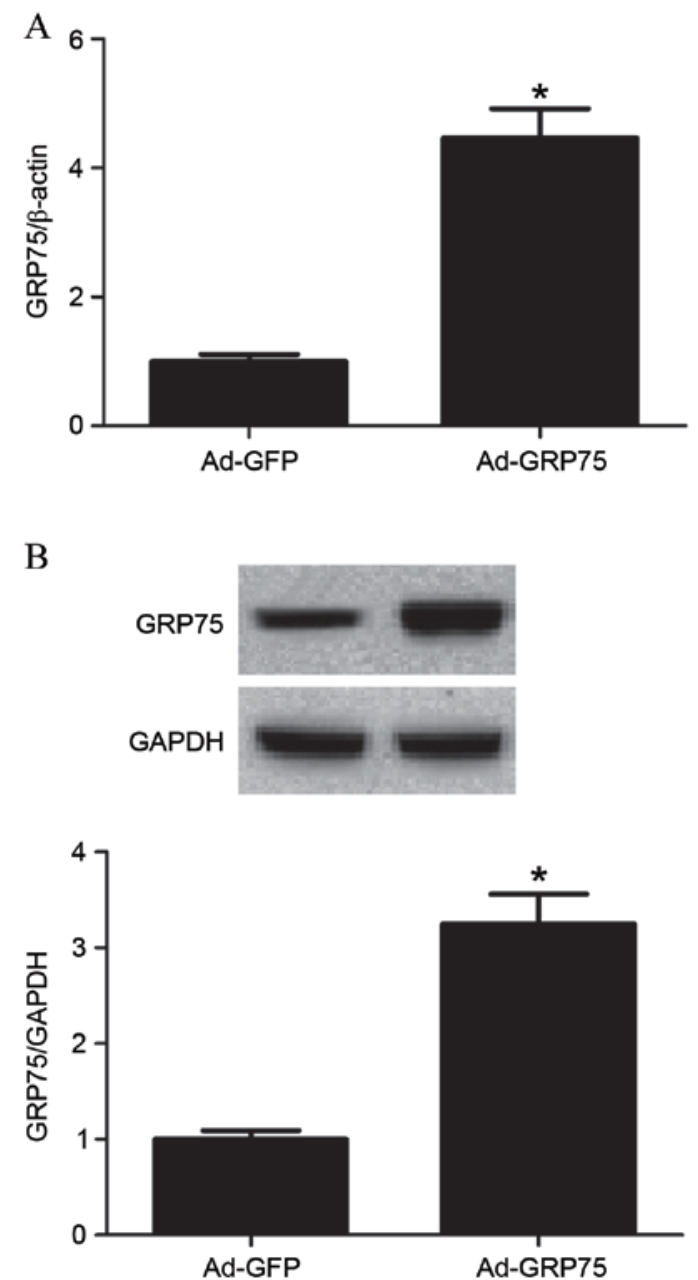

Figure 2. Increased expression of GRP75 in vivo following intrathecal injection of Ad-GRP75. (A) GRP75 mRNA expression levels were detected in brain tissues following ICH by reverse transcription-quantitative polymerase chain reaction. $\beta$-actin was used as a normalizing control. (B) GRP75 protein expression levels in brain tissues following ICH were measured by western blot. Protein expression was normalized to GAPDH. Values are presented as the mean \pm standard deviation. $\mathrm{n}=5$ rats per group. ${ }^{*} \mathrm{P}<0.05$ vs. Ad-GFP group. GRP75, glucose-regulated protein 75; ICH, intracerebral hemorrhage; Ad-, adenoviral overexpression vector; GFP, green fluorescent protein.

ICH significantly upregulated p-Akt levels compared with the $\mathrm{ICH}$ group ( $\mathrm{P}=0.036$; Fig. 5).

\section{Discussion}

In the present study, significantly reduced expression of GRP75 was demonstrated in the brain tissues of rats with ICH. In addition, overexpression of GRP75 in the brain tissues of rats with ICH significantly inhibited the production of inflammatory cytokines and affected production of neuronal apoptosis markers. Furthermore, overexpression of GRP75 significantly upregulated p-Akt in brain tissues following ICH.

GRP75 participates in neuronal processes $(5,11,12)$. It has been reported that the expression of GRP75 decreased significantly in dopaminergic cells overexpressing A53T $\alpha$-synuclein, and downregulation of GRP75 attenuated the disrupted mitochondrial dynamics by reducing $\alpha$-synuclein translocation to mitochondria (13). Liu et al (14) reported that upon exposure to glucose deprivation, GRP75-overexpressing 

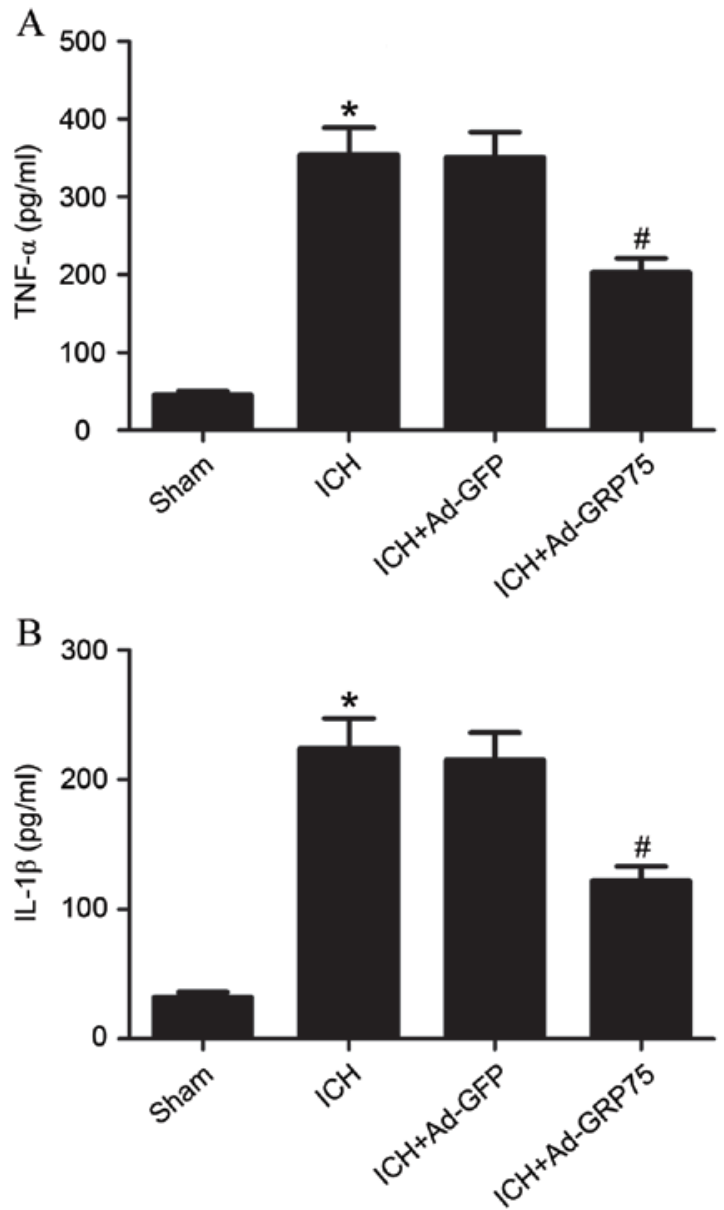

Figure 3. Overexpression of GRP75 inhibits inflammation in a rat model of ICH. The concentrations of (A) TNF- $\alpha$ and (B) IL-1 $\beta$ in brain tissues 2 days following ICH were analyzed by ELISA. Values are presented as the mean \pm standard deviation. $\mathrm{n}=5$ rats per group. ${ }^{*} \mathrm{P}<0.05$ vs. sham group; ${ }^{\#} \mathrm{P}<0.05$ vs. ICH group. GRP75, glucose-regulated protein 75 ; ICH, intracerebral hemorrhage; TNF- $\alpha$, tumor necrosis factor- $\alpha$; IL-1 $\beta$, interleukin- $1 \beta$; Ad-, adenoviral overexpression vector; GFP, green fluorescent protein.

PC12 cells exhibited more moderate cell damage than control PC12 cells. Consistent with these results, the present study demonstrated reduced GRP75 expression in brain tissues following ICH. These data suggest that GRP75 may be important in the progression of ICH.

Activation of the inflammatory cascade is a common event in the ICH-induced brain (15). Previous studies have reported that inflammatory cytokines, including TNF- $\alpha$ and IL-1 $\beta$, were upregulated following ICH (16-18). In the present study, overexpression of GRP75 was observed to significantly suppress the production of TNF- $\alpha$ and IL- $1 \beta$ in the brain tissues of rats with ICH.

Neuronal apoptosis is important in the progression of ICH (2). Caspase-3, known as the death enzyme, has is important in the controlled execution of programmed cell death (19). In the present study, overexpression of GRP75 was demonstrated to significantly inhibit the expression of active caspase-3. The apoptotic protein Bax is a Bcl-2 family cytoplasmic protein, which, following activation by diverse stimuli, inserts into mitochondria, leading to caspase-3 activation and cell apoptosis (20). In the present study, overexpression of GRP75 was observed to significantly decrease the expression
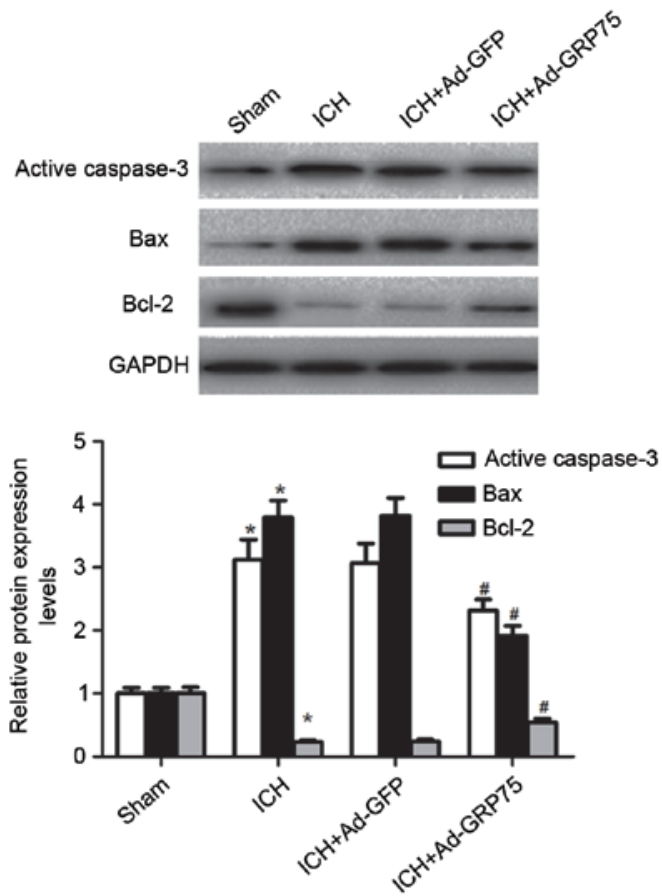

Figure 4. Overexpression of GRP75 reduces expression of neuron apoptosis markers in a rat model of ICH. Western blot analysis of active caspase-3, Bax and $\mathrm{Bcl}-2$ protein levels in brain tissues 2 days following $\mathrm{ICH}$, with quantification relative to GAPDH. Values are presented as the mean \pm standard deviation. $\mathrm{n}=5$ rats per group. ${ }^{~} \mathrm{P}<0.05$ vs. sham group; ${ }^{~} \mathrm{P}<0.05$ vs. $\mathrm{ICH}$ group. GRP75, glucose-regulated protein 75; ICH, intracerebral hemorrhage; Bax, $\mathrm{Bcl}-2$ associated $\mathrm{X}$ apoptosis regulator; Bcl-2, B cell lymphoma 2 apoptosis regulator; Ad-, adenoviral overexpression vector; GFP, green fluorescent protein.
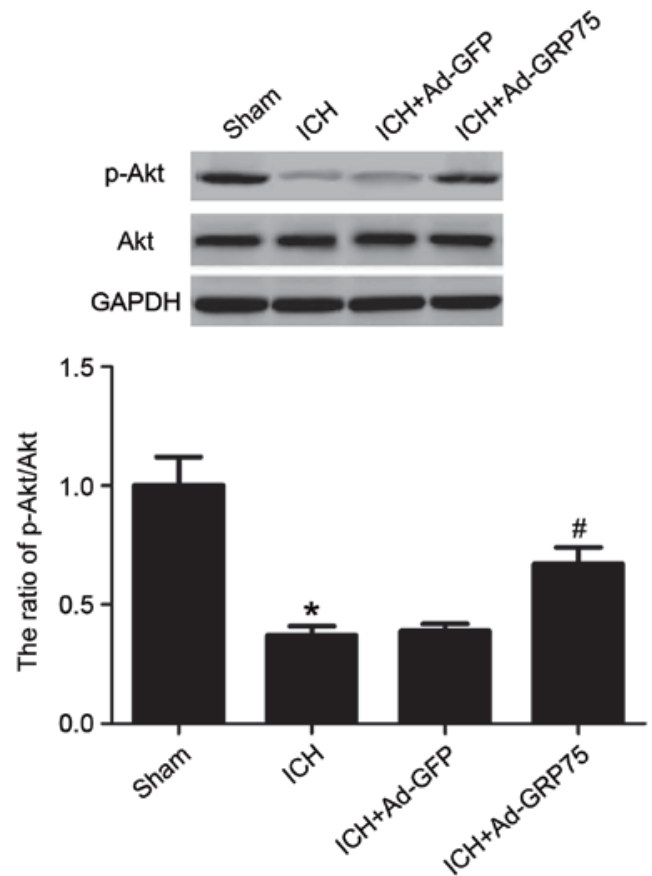

Figure 5. Overexpression of GRP75 upregulates the level of p-Akt in a rat model of ICH. Western blot analysis of p-Akt and Akt expression levels in brain tissues at 2 days after $\mathrm{ICH}$, with quantification relative to GAPDH. Values are presented as the mean \pm standard deviation of the ratio of p-Akt to total Akt. $\mathrm{n}=5$ rats per group. ${ }^{*} \mathrm{P}<0.05$ vs. sham group; ${ }^{*} \mathrm{P}<0.05$ vs. $\mathrm{ICH}$ group. GRP75, glucose-regulated protein 75 ; ICH, intracerebral hemorrhage; p-, phosphorylated; Ad-, adenoviral overexpression vector; GFP, green fluorescent protein. 
of Bax and increase the expression of Bcl-2 in brain tissues of rats with $\mathrm{ICH}$.

Previous studies have demonstrated that the PI3K/Akt pathway is an important signal transduction pathway that regulates cell survival of neurons (21-23). Various cellular stresses can activate Akt through phosphorylation, which phosphorylates a range of Akt downstream targets such as Bcl-2-associated death promoter (Bad), glycogen synthase kinase-3 beta (GSK3 $\beta$ ) and forkhead box O-subclass (FoxO) proteins, leading to the survival of target cells $(24,25)$. Previous studies demonstrated that Akt inhibits apoptosis by phosphorylating and inactivating pro-apoptotic factors (such as Bad) to maintain mitochondrial integrity through preventing the inhibition of anti-apoptotic Bcl-2 by Bad (26); Yang et al (27) demonstrated that the activation of Akt by GRP75 inhibited the Bax conformational change and, subsequently, apoptosis. The present study observed that the level of p-Akt was significantly downregulated in brain tissues following $\mathrm{ICH}$, and overexpression of GRP75 upregulates the level of p-Akt. These results indicated that GRP75 promotes neuronal apoptosis following ICH through modulation of the Akt-dependent pathway.

In conclusion, the present study demonstrated for the first time (to the best of our knowledge) that GRP75 expression is significantly decreased in brain tissues following $\mathrm{ICH}$, and that overexpression of GRP75 inhibits inflammation and neuronal apoptosis in a rat model of ICH. Therefore, GR P75 may represent a promising target for the treatment of $\mathrm{ICH}$.

\section{References}

1. Schmidt LB, Geertz S, Wohlfahrt J, Melbye M and Munch TN Recurrent intracerebral hemorrhage: Associations with comorbidities and medicine with antithrombotic effects. PLoS One 11: e0166223, 2016.

2. Matsushita K, Meng W, Wang X, Asahi M, Asahi K, Moskowitz MA and Lo EH: Evidence for apoptosis after intracerebral hemorrhage in rat striatum. J Cereb Blood Flow Metab 20: 396-404, 2000.

3. Ran Q, Wadhwa R, Kawai R, Kaul SC, Sifers RN, Bick RJ, Smith JR and Pereira-Smith OM: Extramitochondrial localization of mortalin/mthsp70/PBP74/GRP75. Biochem Biophys Res Commun 275: 174-179, 2000

4. Wadhwa R, Taira K and Kaul SC: An Hsp70 family chaperone, mortalin/mthsp70/PBP74/Grp75: What, when, and where? Cell Stress Chaperones 7: 309-316, 2002.

5. Zhang G, Han M, Wang X and Xiao A: GRP75 Involves in retinal ganglion cell apoptosis after rat optic nerve crush. J Mol Neurosci 56: 422-430, 2015.

6. Yang L, Liu X, Hao J, Yang Y, Zhao M, Zuo J and Liu W: Glucose-regulated protein 75 suppresses apoptosis induced by glucose deprivation in PC12 cells through inhibition of Bax conformational change. Acta Biochim Biophys Sin (Shanghai) 40: 339-348, 2008

7. Voloboueva LA, Emery JF, Sun X and Giffard RG: Inflammatory response of microglial BV-2 cells includes a glycolytic shift and is modulated by mitochondrial glucose-regulated protein 75/mortalin. FEBS Lett 587: 756-762, 2013.

8. Yang S, Song S, Hua Y, Nakamura T, Keep RF and Xi G: Effects of thrombin on neurogenesis after intracerebral hemorrhage. Stroke 39: 2079-2084, 2008.

9. Livak K and Schmittgen T: Analysis of relative gene expression data using real-time quantitative PCR and the 2(-Delta Delta C(T)) Method. Methods 25: 402-408, 2001.
10. Tao X, Xie L, Duan C, Dai S, Ren J, Yan Y, Shen J, Lu H and $\mathrm{Ge} \mathrm{J}$ : Up-regulation of interferon regulatory factor 3 involves in neuronal apoptosis after intracerebral hemorrhage in adult rats. Neurochem Res 41: 2937-2947, 2016.

11. Shih YY, Lee H, Nakagawara A, Juan HF, Jeng YM, Tsay YG, Lin DT, Hsieh FJ, Pan CY, Hsu WM and Liao YF: Nuclear GRP75 binds retinoic acid receptors to promote neuronal differentiation of neuroblastoma. PLoS One 6: e26236, 2011.

12. Jin J, Hulette C, Wang Y, Zhang T, Pan C, Wadhwa R and Zhang J: Proteomic identification of a stress protein, mortalin/mthsp70/GRP75: Relevance to Parkinson disease. Mol Cell Proteomics 5: 1193-1204, 2006.

13. Liu Ft Chen Y, Yang Yj, Yang L, Yu M, Zhao J, Wu JJ, Huang F, Liu W, Ding ZT and Wang J: Involvement of mortalin/GRP75/mthsp70 in the mitochondrial impairments induced by A53T mutant $\alpha$-synuclein. Brain Res 1604: 52-61, 2015.

14. Liu Y, Liu W, Song XD and Zuo J: Effect of GRP75/mthsp70/PBP74/mortalin overexpression on intracellular ATP level, mitochondrial membrane potential and ROS accumulation following glucose deprivation in PC12 cells. Mol Cell Biochem 268: 45-51, 2005.

15. Wu J, Yang S, Xi G, Song S, Fu G, Keep RF and Hua Y: Microglial activation and brain injury after intracerebral hemorrhage. Acta Neurochir Suppl 105: 59-65, 2008.

16. Wu S, Fang CX, Kim J and Ren J: Enhanced pulmonary inflammation following experimental intracerebral hemorrhage. Exp Neurol 200: 245-249, 2006

17. Hirashima Y, Nakamura S, Endo S, Kuwayama N, Naruse Y and Takaku A: Elevation of platelet activating factor, inflammatory cytokines, and coagulation factors in the internal jugular vein of patients with subarachnoid hemorrhage. Neurochem Res 22: 1249-1255, 1997.

18. Abraham E, Bursten S, Shenkar R, Allbee J, Tuder R, Woodson P, Guidot DM, Rice G, Singer JW and Repine JE: Phosphatidic acid signaling mediates lung cytokine expression and lung inflammatory injury after hemorrhage in mice. J Exp Med 181: 569-575, 1995.

19. Tawa P, Hell K, Giroux A, Grimm E, Han Y, Nicholson DW and Xanthoudakis S: Catalytic activity of caspase-3 is required for its degradation: Stabilization of the active complex by synthetic inhibitors. Cell Death Differ 11: 439-447, 2004.

20. Smaili SS, Hsu YT, Sanders KM, Russell JT and Youle RJ: Bax translocation to mitochondria subsequent to a rapid loss of mitochondrial membrane potential. Cell Death Differ 8: 909-920, 2001.

21. Leinninger GM, Backus C, Uhler MD, Lentz SI and Feldman EL: Phosphatidylinositol 3-kinase and Akt effectors mediate insulin-like growth factor-I neuroprotection in dorsal root ganglia neurons. FASEB J 18: 1544-1546, 2004.

22. Xu X, Chua CC, Gao J, Chua KW, Wang H, Hamdy RC and Chua BH: Neuroprotective effect of humanin on cerebral ischemia/reperfusion injury is mediated by a PI3K/Akt pathway. Brain Res 1227: 12-18, 2008

23. Brunet A, Datta SR and Greenberg ME: Transcription-dependent and-independent control of neuronal survival by the PI3K-Akt signaling pathway. Curr Opin Neurobiol 11: 297-305, 2001.

24. Romashkova JA and Makarov SS: NF- $\mathrm{B}$ is a target of AKT in anti-apoptotic PDGF signalling. Nature 401: 86-90, 1999.

25. Zheng WH, Kar S and Quirion R: Insulin-like growth factor-1-induced phosphorylation of transcription factor FKHRL1 is mediated by phosphatidylinositol 3-kinase/akt kinase and role of this pathway in insulin-like growth factor-1-induced survival of cultured hippocampal neurons. Mol Pharmacol 62: 225-233, 2002.

26. Zhang X, Chen Y, Jenkins LW, Kochanek PM and Clark RS: Bench-to-bedside review: Apoptosis/programmed cell death triggered by traumatic brain injury. Crit Care 9: 66-75, 2005.

27. Yang L, Guo W, Zhang Q, Li H, Liu X, Yang Y, Zuo J and Liu W: Crosstalk between Raf/MEK/ERK and PI3K/AKT in suppression of Bax conformational change by Grp75 under glucose deprivation conditions. J Mol Biol 414: 654-666, 2011. 